\title{
Variación individual e indicios de variación geográfica en el canto territorial del chucao (Scelorchilus rubecula) en el centro-sur de Chile
}

\section{Individual variation and potential geographic variation in the territorial song of Chucao tapaculo (Scelorchilus rubecula) in south-central Chile}

\section{Heraldo V. Norambuena ${ }^{1,2^{*}}$ \& Pedro F. Victoriano ${ }^{1}$}

\author{
${ }^{1}$ Departamento de Zoología, Facultad de Ciencias Naturales y Oceanográficas, Universidad de Concepción, Casilla 160-C, Concepción, Chile. \\ ${ }^{2}$ Centro de Estudios Agrarios y Ambientales, Casilla 164, Valdivia, Chile. \\ *E-mail: buteonis@gmail.com
}

\section{RESUMEN}

Un paradigma en ornitología sostiene que en los Passeriformes subóscinos (suborden Tyranni) los cantos son heredados genéticamente $y$, generalmente, no muestran ninguna evidencia de aprendizaje vocal y variación individual o geográfica. En este trabajo se evalúa la variación individual y geográfica en las vocalizaciones territoriales de cinco poblaciones de Scelorchilus rubecula, un ave suboscina endémica de los bosques templados del sur de Argentina y Chile. Se utilizaron tres métodos complementarios (1) correlación cruzada de sonogramas, (2) coeficiente de variación y (3) ordenamiento multidimensional (MDS). Los resultados sugieren consistencia en las vocalizaciones a nivel individual y variación vocal entre individuos de $S$. rubecula. Esta alta consistencia de las vocalizaciones individuales (66 - $91 \%$ ) en relación con las vocalizaciones de otros individuos de la misma población (17 - $50 \%$ ), indican que algunas variables (i.e., duración frase 1, FMA inicio frase 1, FMA final frase 2) de la vocalización territorial de $S$. rubecula pueden ser utilizadas para la identificación individual, mientras que a nivel geográfico/poblacional no se obtuvo una clara estructuración, áreas geográficas distantes se agruparon en los arreglos MDS.

Palabras clave: cantos, comunicación vocal, subóscinos, variación vocal.

\section{ABSTRACT}

A paradigm in ornithology argues that in suboscine Passerines (suborder Tyranni) songs are genetically inherited and usually do not show evidence of vocal learning and individual or geographic variation. In this work, we evaluated the individual and geographic variation in the territorial songs of five populations of Scelorchilus rubecula, a suboscine bird endemic of the temperate forests of southern Argentina and Chile. Three complementary methods were used (1) sonograms cross-correlation, (2) coefficient of variation and (3) non-metric multi-dimensional scaling (MDS). The results suggest individually consistency in songs and vocal variation among individuals of $S$. rubecula. This high consistency of individual songs $(66-91 \%)$ in comparison with songs of other individuals of the same population (17 - 50 \%) indicate that some variables (i.e., duration of phrase 1, FMA start phrase 1 and FMA end phrase 2) of $S$. rubecula territorial song can be used for individual identification. At geographical/population-level the MDS analysis did not show a clear structured pattern, distant geographic areas were grouped in the MDS arrangements.

Keywords: songs, suboscines, vocal communication, vocal variation. 


\section{INTRODUCCIÓN}

Las aves del orden Passeriformes, se dividen en dos grandes subórdenes: Passeri, comúnmente conocidas como las aves cantoras oscinas y Tyranni, conocidas como las aves cantoras suboscinas (Sibley \& Alquist 1990; Barker et al. 2002). Un paradigma que ha perdurado por bastante tiempo en ornitología sostiene que en los oscinos el aprendizaje del canto tiene un componente cultural (véase Beecher \& Brenowitz 2005 para una revisión), mientras que en los subóscinos los cantos son heredados genéticamente $y$, generalmente, no muestran ninguna evidencia de aprendizaje vocal (Kroodsma 1984, 1985, 1989). Sin embargo, estudios recientes han revelado que el canto en subóscinos puede ser más variable y complejo de lo que se pensaba. Esta variabilidad se puede identificar en dos niveles, uno individual, en donde las vocalizaciones son utilizadas por machos (e.g., Lovell \& Lein 2004), y en casos particulares además por las hembras (Seddon \& Tobias 2010; Odom \& Benedict 2018), para reconocer a sus conespecíficos; y un nivel geográfico, donde se han identificado variaciones en la estructura de las vocalizaciones entre poblaciones de una misma especie (Lindell 1998; Sedgwick 2001; Leger \& Mountjoy 2003; Kroodsma 2005; Seddon \& Tobias 2007; Fitzsimmons et al. 2008, 2011, Ippi et al. 2011, Foote et al. 2013). Esta variabilidad puede ocurrir simultáneamente en una especie, por ejemplo, Fitzsimmons et al. $(2008,2011)$ registraron variación tanto a nivel individual como geográfico para el guardabosques gritón (Lipaugus vociferans), mientras que Ippi et al. (2011) solo reportó variación geográfica en los cantos del rayadito (Aphrastura spinicauda), lo que se atribuyó en ambos casos a diferentes presiones ecológicas. A pesar de estos hallazgos, los estudios sobre variación individual y geográfica en subóscinos siguen siendo escasos, en comparación al amplio conocimiento que se tiene sobre los cantos de los oscinos (Beecher \& Brenowitz 2005; Bradbury \& Vehrencamp 2011; Sandoval \& Menill 2014; Sandoval et al. 2014). La descripción de la variación en los cantos de los subóscinos y la comprensión de su significado funcional es un importante primer paso para el estudio de los patrones de comunicación en este grupo, y puede dar lugar a importantes descubrimientos sobre la evolución del comportamiento de aprendizaje y comunicación vocal (Foote et al. 2013). Además, cualquier diferencia inter-poblacional de las vocalizaciones en una especie que no posee aprendizaje vocal, puede sugerir que otros factores están actuando sobre las variaciones vocales (véase Seddon et al. 2002). Por tanto, el estudio de las vocalizaciones en subóscinos que muestran diferencias vocales entre poblaciones podría proporcionar ideas sobre cómo las señales acústicas evolucionan en respuesta a diferentes presiones selectivas (Endler 1992; Foster \& Endler 1999; Boughman 2002; Seddon 2005).

El Chucao Scelorchilus rubecula (Kittlitz, 1830) es un ave suboscino de la familia Rhinocryptidae, endémico de los bosques templados de Argentina y Chile (Ridgely \& Tudor 1994; Krabbe \& Schulenberg 2003). Se distribuye en Chile desde la provincia de Colchagua región de O'Higgins hasta el norte de la Región de Magallanes, y en Argentina en una estrecha franja desde Neuquén hasta Chubut (Ridgely \& Tudor 1994). Es un ave omnívora, que consume insectos y frutos (Correa et al. 1990; Rozzi et al. 1996; Amico \& Aizen 2005), residente, altamente territorial, y su dispersión se ve afectada por la falta de conectividad entre ambientes boscosos (Willson 2004), lo cual sugiere un potencial nivel de aislamiento entre poblaciones. Su repertorio vocal es variado, con unas 8-10 vocalizaciones utilizadas para el marcaje territorial y contacto entre individuos (Norambuena obs. pers.). Su vocalización más frecuente, y de connotaciones territoriales, es la vocalización onomatopéyica descrita como "chuu chu-chu-chu-caoow", una vocalización breve, grave y reverberante (Ridgely \& Tudor 1994; Krabbe \& Schulenberg 2003). El objetivo de este trabajo es evaluar si la vocalización territorial de $S$. rubecula presenta variación a nivel individual y geográfico/poblacional, a través de grabaciones propias y de otros autores obtenidas del repositorio en línea, https:// www.xeno-canto.org/.

\section{MÉTODOS}

VOCALIZACIONES PROPIAS

Se generó una base de datos con vocalizaciones propias y vocalizaciones disponibles en xeno-canto. Las vocalizaciones propias $(\mathrm{N}=15)$ provienen de un individuo de $S$. rubecula grabado en el Parque Nacional (PN) Conguillío (38 $30^{\circ} \mathrm{S}$; $71^{\circ} 30^{\prime} \mathrm{O}$ ), el 30 de enero de 2014 (entre las 10:00 a 12:00 hr) El individuo grabado en el PN Conguillío fue atraído mediante la técnica de «playback» utilizando un reproductor digital conectado a un parlante (8 watts). Las vocalizaciones fueron grabadas con un equipo Tascam DR-60D y un micrófono condensador Sennheiser ME66/K6. Todas las grabaciones fueron realizadas en formato WAV, a 16 bits con una frecuencia de muestreo de $44,1 \mathrm{kHz}$. Aunque la movilidad de $S$. rubecula se ve afectada principalmente por la fragmentación (Wilson 2004), dada la escasa capacidad de dispersión de $S$. rubecula y la gran distancia y desconexión entre localidades (> $140 \mathrm{~km}$ ), las grabaciones de cada localidad son consideradas como poblaciones distintas. 


\section{VOCALIZACIONES DE XENO-CANTO}

La base de cantos se complementó con grabaciones de otras cuatro localidades disponibles en la base de datos acústica xeno-canto (https://www.xeno-canto.org/) las que se descargaron el 30 de octubre de 2016. De un total de 100 grabaciones disponibles en xeno-canto, se utilizaron 52 por presentar la relación señal-ruido más alta (i.e. diferencia entre nivel de señal y nivel de ruido de fondo (Ls-Ln) inferior a 15 dBA); de éstas 15 correspondieron a un individuo del PN Conguillío, 10 a cuatro individuos del PN Lanín en Argentina (40 $09^{\circ} \mathrm{S}$; $\left.71^{\circ} 21^{\prime} \mathrm{O}\right), 17$ a cinco individuos de la

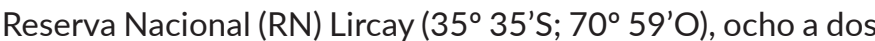
individuos de los Nevados de Chillán (36 $52^{\circ}$ 'S; $71^{\circ} 26^{\prime} \mathrm{O}$ ) y dos a un individuo del PN Lago Puelo (42 ${ }^{\circ} 11^{\prime} \mathrm{S} ; 71^{\circ} 41^{\prime} \mathrm{O}$; véase Apéndice 1). El $68 \%$ de las grabaciones fue solicitada en formato.WAV a los autores y el resto fue transformada a .WAV con el programa Adobe Audition CS6 (2012).

\section{ANÁLISIS DE LAS VOCALIZACIONES}

Las mediciones a escala fina y los sonogramas (i.e., representación gráfica de la frecuencia en función del tiempo) se realizaron con el programa Raven Pro 1.4 (Bioacoustics Research Program 2011), usando los parámetros del espectrograma por defecto (Window-Type: Hann, tamaño: 256 muestras $=5.33 \mathrm{~ms}$ ), $3 \mathrm{~dB}$ filtro ancho de banda: $270 \mathrm{~Hz}$; superposición cuadrícula de tiempo: $50 \%$, tamaño de salto: 128 muestras (=2.67 ms); frecuencia cuadrícula -DFT: 256 muestras, espaciado cuadrícula: $188 \mathrm{~Hz}$. Se aplicó un filtro de banda para eliminar el ruido fuera de la gama de frecuencias de la vocalización de Scelorchilus rubecula, eliminando el ruido por debajo de $200 \mathrm{~Hz}$ y por encima de $3000 \mathrm{~Hz}$. Las variables medidas en cada sonograma fueron: (i) duración de la frase 1 (seg), (ii) duración de la frase 2 (seg), (iii) duración de la frase 3 (seg), (iv) duración total de la vocalización (seg), (v) frecuencia máxima amplitud (FMA) del inicio de la frase $1(\mathrm{~Hz})$, (vi) FMA final de la frase $1(\mathrm{~Hz})$, (vii) ancho de banda de la frase 1 (f2-f1), (viii) FMA inicio de la frase $2(\mathrm{~Hz})$, (ix) FMA final de la frase $2(\mathrm{~Hz}),(\mathrm{x})$ ancho de banda de la frase 2 (f4-f3), (xi) FMA inicio de la frase 3 (Hz), (xii) FMA final de la frase $3(\mathrm{~Hz})$, (xiii) ancho de banda de la frase 3 (f6-f5), (xiv) número de notas de la frase 2 y (xv) número de notas de la frase 3 (ver Fig. 1).

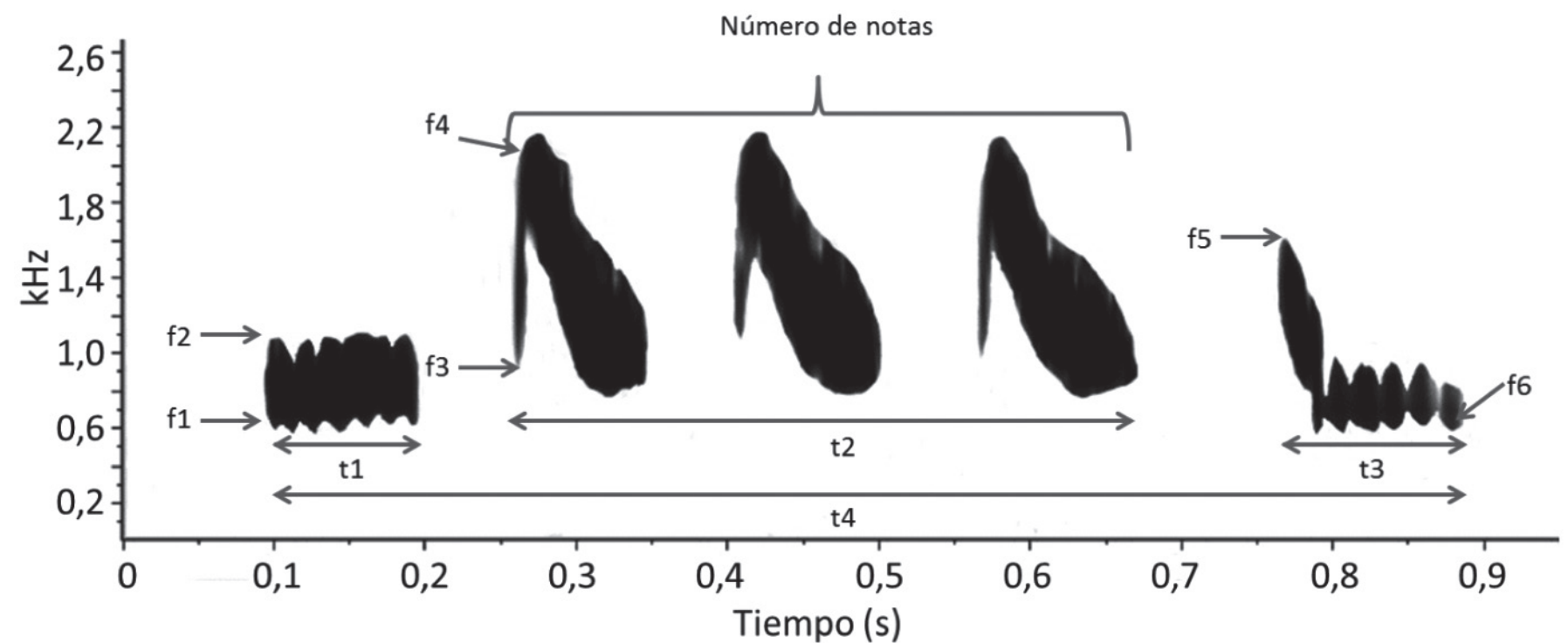

Figura 1. Sonograma del canto territorial de Scelorchilus rubecula, se muestran las medidas a escala fina: duración frase 1 (t1), duración frase 2 (t2), duración frase 3 (t3), duración total (t4), FMA inicio frase 1 (f1), FMA final frase 1 (f2), ancho de banda frase 1 (f2-f1), FMA inicio frase 2 (f3), FMA final frase 2 (f4), ancho de banda frase 2 (f4-f3), FMA inicio frase 3 (f5), FMA final frase 3 (f6), ancho de banda frase 3 (f6-f5), número de notas frase 2 y número de notas frase 3 . / Sonogram of territorial vocalization of Scelorchilus rubecula, the fine-scale measurements are shown: duration phrase 1 (t1), duration phrase 2 (t2), duration phrase 3 (t3), total duration (t4), FMA beginning phrase 1 ( $f 1$ ), FMA end phrase 1 (f2), bandwidth phrase 1 (f2-f1), FMA beginning phrase 2 (f3), FMA end phrase 2 ( $f 4$ ), bandwidth phrase 2 ( $f 4-f 3$ ), FMA beginning phrase 3 (f5), FMA end phrase 3 (f6), bandwidth phrase 3 ( $f 6-f 5$ ), number of notes phrase 2 and number of notes phrase 3 . 
ANÁLISIS DE DATOS

Se utilizaron tres métodos para evaluar la variación de las vocalizaciones en el plano individual y poblacional.

Correlación cruzada de sonogramas.- El primer método de clasificación utilizado fue la correlación cruzada de sonogramas, utilizando el programa Raven Pro 1.4 (Bioacoustics Research Program 2011). La correlación cruzada de sonogramas consiste en analizar el sonograma de cada individuo y calcular el porcentaje de solapamiento con cada sonograma de las vocalizaciones del mismo individuo, o de todos los demás individuos, siguiendo la fórmula:

$$
C_{\Delta t}=\frac{\sum_{t=1 f=1}^{n} \sum_{t, f}^{F F T}\left(X_{t, \Delta t, f}\right)}{\sqrt{\left(\sum_{t=1 f=1}^{n} \sum_{t=1}\left(X_{t, f}\right)^{2}\right)\left(\sum_{t=1 f=1}^{n} \sum_{t, f}\left(Y_{t, f}\right)^{2}\right)}}
$$

donde $n$ es igual $\left(N_{1}+N_{2}\right)$ - 1 y $N_{1}$ y $N_{2}$ son el número de parámetros en el sonograma. FFT es el número de puntos de frecuencia de cada sonograma, los que deben ser los mismos para que los dos sonogramas se correlacionen. $\mathrm{X}_{\mathrm{t}, \mathrm{f}} \mathrm{y}_{\mathrm{t}, \mathrm{t}, \mathrm{f}}$ son los valores de amplitud (en este caso, la energía del sonograma o FMA) en los dos sonogramas en una frecuencia $f$, y tiempo $t$, y $t+\Delta t$, respectivamente. Los valores de correlación normalizada varían entre 0 y 1 , donde 0 significa que los valores en los dos sonogramas no coinciden en absoluto, mientras que una correlación de 1 indica que los dos sonogramas son idénticos, este valor fue transformado a porcentaje para facilitar su interpretación. Este análisis se utilizó para comparaciones intra-individuales, intra-poblacionales y/o inter-poblacionales. Los resultados se muestran como media $\pm \mathrm{DE}$.

Coeficiente de variación.- Se calculó la media \pm DE de los 15 caracteres estructurales finos para las vocalizaciones de cada individuo. Se utilizaron coeficientes de variación (CV) para cuantificar la magnitud de la variabilidad para cada carácter de la vocalización. Se calcularon coeficientes de variación individual $\left(\mathrm{CV}_{\mathrm{a}}\right)$ y coeficientes de variación entre individuos $\left(\mathrm{CV}_{\mathrm{b}}\right)$ de cada población. Sólo se consideraron los individuos con $>2$ vocalizaciones, y se excluyeron del cálculo de $\mathrm{CV}_{\mathrm{b}}$ las frases ausentes de alguna de las vocalizaciones. Las relaciones intra-individuos y entre individuos fueron usadas para calcular el coeficiente de variación $\mathrm{CV}_{w}$, esta es una medida que relaciona la variabilidad y se calcula como $\mathrm{CV}_{\mathrm{a}}$ / $\mathrm{CV}_{\mathrm{b}}$. Cuando se obtiene una relación $\mathrm{CV}_{\mathrm{w}}>1,0$ significa que la variable del sonograma o vocalización es más variable entre individuos que individualmente, esto se ha sugerido como una señal de identidad individual (Fitzsimmons et al. 2008).

Análisis MDS y Anosim.- Con el objetivo de determinar si las vocalizaciones de individuos y poblaciones pueden clasificarse sobre la base de mediciones estructurales finas, se realizó un análisis de similitud basado en distancia Euclidiana con el programa Primer v.6.0 (Clarke 1993). A partir de las hemimatrices de similitud, se obtuvo un arreglo mediante un análisis multivariado no paramétrico de escalamiento multidimensional (MDS; Kruskal \& Wish 1978), para evaluar y visualizar los arreglos de similitud entre áreas de muestreo. Los arreglos basados en similitud fueron usados para evaluar significancia estadística de la formación de grupos, la que se estimó mediante un análisis de similitud (ANOSIM; Clarke 1993) en el programa Primer v.6.0. Los datos son presentados como media $\pm \mathrm{DE}$.

\section{RESULTADOS}

VARIACIÓN INDIVIDUAL EN LA VOCALIZACIÓN

Los resultados del análisis de correlación cruzada de sonogramas a nivel intra-individual mostraron una alta correlación en las vocalizaciones en el PN Conguillío (74,97 $\pm 10,87 \%)$, PN Lanín (66,18 $\pm 19,71 \%)$, PN Lircay (82,84 \pm $16,34 \%$ y yevados de Chillán (91,26 $\pm 6,56 \%$ ) los resultados fueron significativamente mayores individualmente que entre individuos $(U=82,84-1.175 p<0,0001)$. A nivel intrapoblacional la correlación entre vocalizaciones fue baja, para el PN Lanín (31,38 \pm 9,56\%), PN Lircay $(50,57 \pm 16,34 \%)$ y Nevados de Chillán (17,81 $\pm 6,13 \%)$.

Todas las características evaluadas en las vocalizaciones mostraron valores $\mathrm{CV}_{w}$ sobre 1,0 , una obtuvo valor $1-2$, seis obtuvieron valor de 2-3 y siete obtuvieron valor 3-4 (Tabla 1). Además, cuatro características (i.e., duración frase 1, FMA inicio frase 1, FMA final frase 2 y número de notas) mostraron valores $>4,0$, por lo que probablemente sean importantes para el reconocimiento individual.

\section{VARIACIÓN GEOGRÁFICA}

Las vocalizaciones entre poblaciones mostraron diferencias estructurales en la composición de las frases 2 y 3 (Fig. 2), en las poblaciones del PN Lanín y Puelo hay un aumento en 

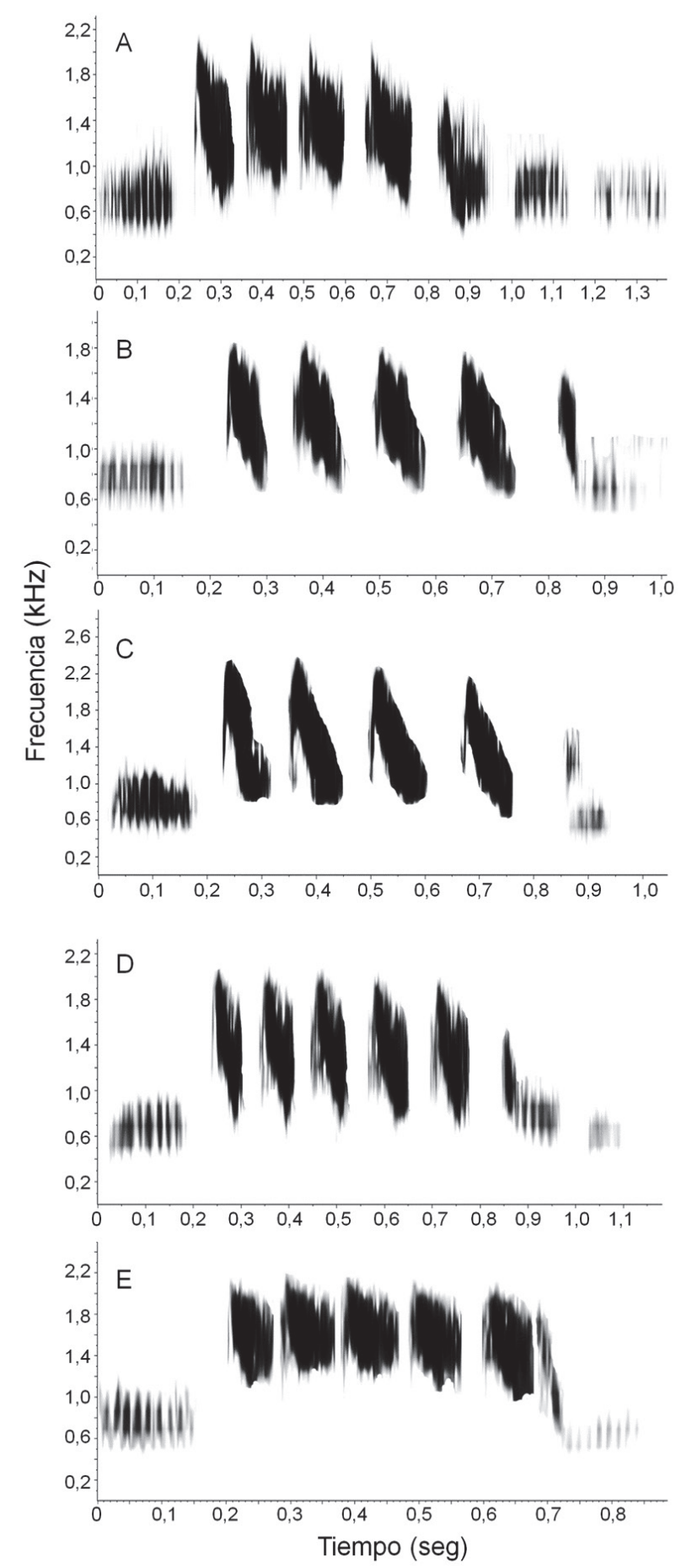

Figura 2. Canto territorial de cinco machos de Scelorchilus rubecula de cinco poblaciones: (A) RN Lircay, (B) Nevados Chillán, (C) PN Conguillío, (D) PN Lanín y (E) PN Lago Puelo. / Territorial songs of five males of Scelorchilus rubecula from five populations: (A) RN Lircay, (B) Nevados Chillan, (C) PN Conguillio, (D) PN Lanin and (E) PN Lago Puelo. el número de sílabas de la frase 2 (5 sílabas), en las otras tres poblaciones esa frase está compuesta sólo por cuatro sílabas. Además, dos poblaciones, RN Lircay y PN Lanín, presentaron un mayor número de sílabas en la frase 3 o terminal, con tres y dos sílabas respectivamente (Fig. 2). Los resultados de correlación cruzada, al comparar la vocalización de un individuo con el resto de los individuos de la misma población $(43,02 \pm 26,62 \%)$, fueron levemente mayores que los resultados entre poblaciones $(29,46 \pm 17,78 \%)$, sin embargo, esta diferencia no fue significativa (Mann-Whitney U-test: $U=435,0 \mathrm{p}<0,147)$. En el análisis de MDS se muestran a lo menos tres grupos, Puelo-Chillán, y dos grupos disimiles que incluyen una combinación de ejemplares de Lircay y Lanín (Fig. 3). Al evaluar la similitud entre poblaciones con el análisis de ANOSIM, se registraron diferencias significativas entre Lanín-Lircay, Lanín-Chillán, Lircay-Chillán y LircayPuelo (Tabla 2), sin embargo, áreas geográficamente lejanas como Lanín-Puelo y Chillán-Puelo no mostraron diferencias significativas (Tabla 2).

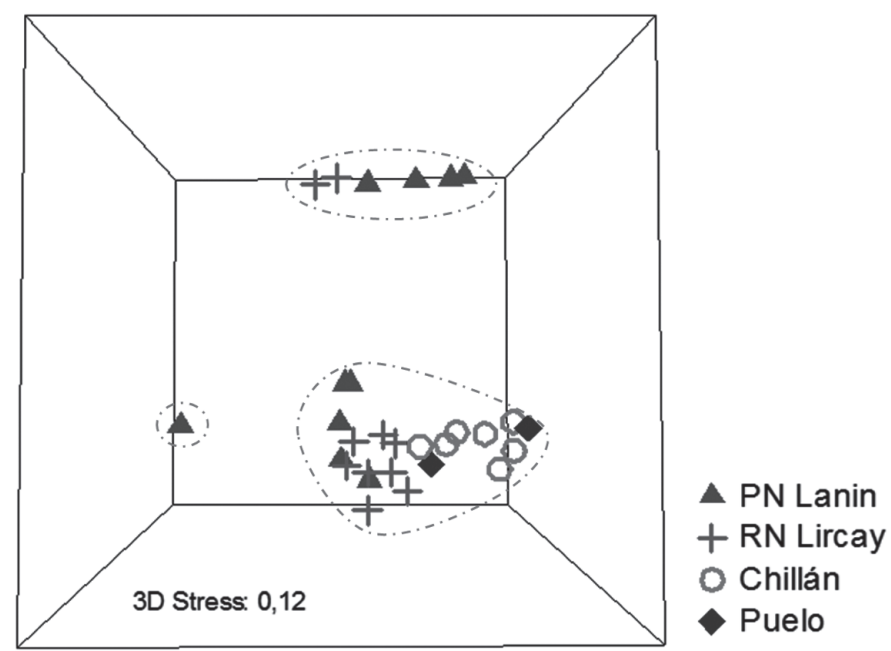

FIgURA 3. Ordenamiento multidimensional (MDS) del canto territorial del chucao (Scelorchilus rubecula) entre cuatro localidades, basado en distancia Euclidiana (Stress: 0,12). Elipses muestran distancia < 25 \%. / Multidimensional scaling (MDS) of the territorial song of Chucao Tapaculo (Scelorchilus rubecula) between four localities, based on Euclidean distance (Stress: 0.12). Ellipses show a distance $<25 \%$. 
TABLA 1. Características estructurales finas del canto territorial de Scelorchilus rubecula. Coeficientes de variación individual (CV), coeficientes de variación entre individuos $\left(\mathrm{CV}_{b}\right)$ de cada población y coeficiente de variación $\mathrm{CVw}_{\mathrm{w}}\left(\mathrm{CV}_{\mathrm{a}} / \mathrm{CV}_{\mathrm{b}}\right)$. / Fine structural characteristics of the territorial song of Scelorchilus rubecula. Individual coefficients of variation (CVa), coefficients of variation between individuals (CVb) of each population and coefficient of variation CVw (CVa / CVb).

\begin{tabular}{lrrrrr}
\hline Variable & Promedio & \multicolumn{1}{c}{ D.E. } & \multicolumn{1}{c}{$\mathrm{CV}_{\mathrm{a}}$} & $\mathrm{CV}_{\mathrm{b}}$ promedio & $\mathrm{CV}_{\mathrm{w}}$ \\
\hline Duración frase 1 (seg) & 0,14 & 0,05 & 31,18 & 7,72 & 4,04 \\
Duración frase 2 (seg) & 0,55 & 0,10 & 6,98 & 5,63 & 1,24 \\
Duración frase 3 (seg) & 0,25 & 0,13 & 47,48 & 18,52 & 2,56 \\
Duración total (seg) & 1,06 & 0,22 & 19,29 & 6,31 & 3,06 \\
FMA inicio de la frase 1 (Hz) & 491,49 & 195,08 & 31,66 & 6,96 & 4,55 \\
FMA final de la frase 1 (Hz) & 896,16 & 334,34 & 28,88 & 4,05 & 7,13 \\
Ancho banda f1-f2 (Hz) & 330,89 & 142,50 & 35,88 & 9,90 & 3,62 \\
FMA inicio de la frase 2 (Hz) & 1169,22 & 174,28 & 14,46 & 5,18 & 2,79 \\
FMA final de la frase 2 (Hz) & 2101,49 & 179,54 & 8,83 & 2,65 & 3,33 \\
Ancho banda f3-f4 (Hz) & 545,86 & 234,35 & 46,29 & 16,65 & 2,78 \\
FMA inicio de la frase 3 (Hz) & 1195,09 & 365,71 & 30,71 & 12,67 & 2,42 \\
FMA final de la frase 3 (Hz) & 566,33 & 120,29 & 20,17 & 9,43 & 2,14 \\
Ancho banda f5-f6 (Hz) & 592,95 & 283,61 & 45,56 & 21,49 & 2,12 \\
Número notas & 4,42 & 0,69 & 16,57 & 3,95 & 4,20 \\
\hline Número notas frase final & 1,50 & 0,67 & 43,04 & 0,00 & - \\
\hline
\end{tabular}

TABLA 2. Análisis de Similaridad (ANOSIM) del canto territorial del chucao (Scelorchilus rubecula) entre cuatro localidades. En negrita valores significativos. / Analysis of Similarity (ANOSIM) of the territorial song of Chucao Tapaculo (Scelorchilus rubecula) between four localities. Significant values in bold.

\begin{tabular}{lcccc}
\hline Grupos & Estadístico R & Nivel de significancia \% & Permutación actual & Número observado \\
\hline PN Lanín, RN Lircay & 0,355 & $\mathbf{0 , 4}$ & 999 & 3 \\
PN Lanín, Chillán & 0,355 & $\mathbf{0 , 6}$ & 999 & 5 \\
PN Lanín, Puelo & 0,115 & 28,8 & 66 & 19 \\
RN Lircay, Chillán & 0,542 & $\mathbf{0 , 1}$ & 999 & 0 \\
RN Lircay, Puelo & 0,511 & $\mathbf{4 , 1}$ & 171 & 7 \\
Chillán, Puelo & 0,047 & 33,3 & 45 & 15 \\
\hline
\end{tabular}

\section{DISCUSIÓN}

Los resultados de dos análisis complementarios (i.e., correlación cruzada y $\mathrm{CV}_{\mathrm{w}}$ ) sugieren consistencia en las vocalizaciones a nivel individual y variación vocal entre individuos de $S$. rubecula. Esta alta consistencia de las vocalizaciones individuales (>65\%), respecto a la asociación con las vocalizaciones de otros individuos de la misma población, indican que algunas variables de la vocalización territorial de $S$. rubecula pueden ser utilizadas para la identificación individual. Esto ha sido documentado para varias especies de subóscinos (e.g. Lovell \& Lein 2004; Seddon \& Tobias 2010; Fitzsimmons et al. 2008, 2011, Foote et al. 2013). Valores elevados de $\mathrm{CV}_{w}$ permiten identificar qué características son las más conservadas en los cantos (Fitzsimmons et al. 2008); para el caso de S. rubecula, cuatro características (i.e., duración frase 1, FMA inicio frase 1, FMA final frase 2 y número de notas) son las más conservadas y podrían ser útiles en la identificación a nivel individual. En aves subóscinos la variación de las vocalizaciones entre individuos sugieren un rol en el reconocimiento de conespecíficos y por tanto podrían tener un rol en el éxito reproductivo (Foote et al. 2013).

En los análisis de correlación cruzada, MDS y ANOSIM, se registraron resultados contrastantes a escala geográfica/ poblacional, donde algunas poblaciones presentaron 
variación geográfica en el canto (véase Tabla 2), mientras que otras no mostraron una clara asociación geográfica, siendo "vocalmente" más próximas poblaciones geográficamente más lejanas (e.g. PN Lanín y Nevados de Chillán). Si la variación geográfica en las vocalizaciones se debiese a diferencias genéticas, se esperaría que poblaciones más cercanas (geográfica y filogenéticamente) mostraran mayor similitud vocal que entre poblaciones lejanas (Wilkins et al. 2013), siempre que la única barrera que limita el flujo génico sea la distancia geográfica (van Strien et al. 2015). Un patrón similar se esperaría si variaciones morfológicas (intrínsicamente genéticas), como cambios en el tamaño corporal o del pico, produjeran la variación en la señal acústica (Darryberry et al. 2018).

A pesar de que la variación geográfica en las vocalizaciones de subóscinos parece no ser un patrón tan recurrente (e.g. Wilczynski \& Ryan 1999; Foote et al. 2013), podría existir más de un factor que determina la presencia o ausencia de variación (Wilkins et al. 2013). En una etapa inicial la variación puede ser altamente aleatoria en especies con vocalizaciones innatas, debido a que estas especies carecen de aprendizaje vocal y apareamiento asociativo, que podría reforzar los límites del dialecto (Mundinger 1982; Odom \& Menill 2012), lo que explicaría que incluso entre poblaciones geográficamente lejanas se presenten vocalizaciones similares. Por lo tanto, las variaciones detectadas en $S$. rubecula pueden ser el resultado de presiones selectivas, no excluyentes entre sí, tales como las diferencias de hábitat que conducen a la adaptación acústica (Morton 1975; Rothstein \& Fleischer 1987; Tubaro \& Segura 1994; Brown \& Handford 1996; Ippi et al. 2011). A lo anterior, se agrega el posible rol de procesos de selección sexual, donde se ha documentado que la preferencia femenina por una mayor complejidad de la señal acústica podría impulsar la divergencia acústica (e.g. Toews \& Irwin 2008; Irwin et al. 2001; Boul et al. 2007). En oscinos y otras especies que presentan aprendizaje vocal, los cantos a menudo muestran marcados límites de dialectos microgeográficos (Marler \& Tamura 1962; Mundinger 1982). Un análisis que permita poner a prueba cada una de las hipótesis de adaptación acústica (características del hábitat) o selección sexual (mediante experimentos de playback), permitirá precisar que procesos generan la variación individual y la posible variación geográfica en las vocalizaciones territoriales de $S$. rubecula.

\section{AGRADECIMIENTOS}

Agradecemos a Álvaro Jaramillo, Bernabé López-Lanús, Rodrigo Valenzuela, Jong King, Lance Benner, Doug
Knapp, Tomek Tumiel y Fabrice Schmitt por su apoyo con las grabaciones utilizadas en este estudio. Además, agradecemos a Enrique Rodríguez-Serrano y Lucila Moreno por sus comentarios al manuscrito, a Enzo Basso y Amado Villalobos-Leiva por su colaboración en terreno. HVN agradece a la beca de doctorado nacional CONICYT PCHA/ Doctorado Nacional/2013-21130354 y FONDECYTPOSTDOCTORADO 3190618. Agradecemos a la Corporación Nacional Forestal por la autorización de estudio en el SNASPE (N011/2014).

\section{REFERENCIAS}

Amico, G.C., Aizen, M.A. 2005. Dispersión de semillas por aves en un bosque templado de Sudamérica austral: ¿quién dispersa a quién? Ecología Austral 15: 89-100.

Barker, F.K., Barrowclough, G.F., Growth, J.G. 2002. A phylogenetic hypothesis for passerine birds: taxonomic and biogeographic implications of an analysis of nuclear DNA sequence data. Proceedings of the Royal Society of London, Series B 269: 295-308.

Beecher, M., Brenowitz, E. 2005. Functional aspects of song learning in songbirds. Trends in Ecology and Evolution 20: 143-149.

Bioacoustics Research Program. 2011. Raven Pro: Interactive Sound Analysis Software (version 1.4). The Cornell Lab of Ornithology, Ithaca, NY.

Boughman, J.W. 2002. How sensory drive can promote speciation. Trends in Ecology and Evolution 17: 571-577.

Boul, K.E., Funk, W.C., Darst, C.R., Cannatella, D.C., Ryan, M.J. 2007. Sexual selection drives speciation in an Amazonian frog. Proceedings of the Royal Society B: Biological Sciences 274: 399-406

Bradbury, J.W., Vehrencamp, S.L. 2011. Principles of animal communication. Sinauer.

Brown, T.J., Handford, V. 1996. Acoustic signal amplitude patterns: a computer simulation investigation of the acoustic adaptation hypothesis. The Condor 98: 608-623.

Clarke, K.R. 1993. Non-parametric multivariate analyses of changes in community structure. Australian Journal of Ecology 18: 117-143.

Correa, A., Armesto, J.J., Schlatter, R.P., Rozzi, R., Torres-Mura, J.C. 1990. La dieta del chucao (Scelorchilus rubecula), un Passeriforme terrícola endémico del bosque templado húmedo de Sudamérica austral. Revista Chilena de Historia Natural 63: 197-202.

Derryberry, E.P., Seddon, N., Derryberry, G.E., Claramunt, S., Seeholzer, G.F., Brumfield, R.T., Tobias, J.A. 2018. Ecological drivers of song evolution in birds: Disentangling 
the effects of habitat and morphology. Ecology and Evolution 8(3): 1890-1905.

Endler, J. A. 1992. Signals, signal conditions, and the direction of evolution. American Naturalist 139: 125-153.

Fitzsimmons, L.P., Barker, N.K., Mennill, D.J. 2008. Individual variation and lek-based vocal distinctiveness in songs of the Screaming Piha (Lipaugus vociferans), a suboscine songbird. The Auk 125: 908-914.

Fitzsimmons, L.P., Barker, N.K., Mennill, D.J. 2011. Further analysis supports the conclusion that the songs of Screaming Pihas are individually distinctive and bear a lek signature. The Auk 128: 790-792.

Foster, S.A., Endler, J.A. 1999. Geographic Variation in Behavior Perspectives on Evolutionary Mechanisms. Oxford University Press, Oxford.

Foote, J.R., Palazzi, E., Mennill, D.J. 2013. Songs of the Eastern Phoebe, a suboscine songbird, are individually distinctive but do not vary geographically. Bioacoustics 22(2): 137151.

Ippi, S., Vásquez R.A., Van Dongen, W.F.D., Lazzoni, I. 2011. Geographical variation in the vocalizations of the suboscine Thorn-tailed Rayadito Aphrastura spinicauda. Ibis 153: 789-805.

Irwin, D.E., Bensch, S., Price, T.D. 2001. Speciation in a ring. Nature 409: 333-337.

Krabbe, N., Schulenberg, T.S. 2003. Family Rhinocryptidae (Tapaculos). In: del Hoyo, J., Elliott, A., Christie, D.A. (Eds) Handbook of the birds of the world. Volume 8: broadbills to tapaculos. 748-787. Lynx Edicions, Barcelona.

Kroodsma, D.E. 1984. Songs of the Alder Flycatcher (Empidonax alnorum) and Willow Flycatcher (Empidonax traillii) are innate. The Auk 101: 13-24.

Kroodsma, D.E. 1985. Development and use of two song forms by the Eastern Phoebe. Wilson Bulletin 97: 21-29.

Kroodsma, D.E. 1989. Male Eastern phoebes (Sayornis phoebe; Tyrannidae, Passeriformes) fail to imitate songs. Journal of Comparative Psychology 103: 227-232.

Kroodsma, D.E. 2005. The singing life of birds: the art and science of listening to birdsong. Houghton Mifflin, New York.

Kruskal, J.B., Wish, M. 1978. Multidimensional Scaling. Sage University Paper Series on Quantitative Applications in the Social Sciences, No. 07-011. Sage Publications, Newbury Park.

Leger, D.W., Mountjoy, D.J. 2003. Geographic variation in song of the Bright-rumped Attila (Tyrannidae: Attila spacdiceus): implications for species status. The Auk 120: 69-74.

Lindell, C. 1998. Limited geographic variation in the vocalizations of a neotropical Furnariid, Synallaxis albescens. Wilson Bulletin 110: 368-374.

Lovell, S.F., Lein, M. 2004. Neighbor-stranger discrimination by song in a suboscine bird, the alder flycatcher, Empidonax alnorum. Behavioral Ecology 15: 799-804.

Marler, P., Tamura, M. 1962. Song "dialects" in three populations of White-crowned Sparrows. The Condor 64: 368-377.

Morton, E.S. 1975. Ecological sources of selection on avian sounds. American Naturalist 109: 17-34.

Mundinger, P.C. 1982. Microgeographic and macrogeographic variation in the acquired vocalizations of birds. In: Kroodsma, D.E., Miller, E. H. (Eds) Acoustic Communication in Birds, vol. 2: 147-208. Academic Press, New York.

Odom, K.A., Mennill D.J. 2012. Inconsistent geographic variation in the calls and duets of Barred Owls across an area of genetic introgression. The Auk 129: 387-398.

Odom, K.J., Benedict, L. 2018. A call to document female bird songs: Applications for diverse fields. The Auk 135(2): 314-325.

Ridgely, R.S., Tudor, G. 1994. The birds of South America. 2. University of Texas Press, Austin, TX.

Rothstein, S.I., Fleischer, R.C. 1987. Vocal dialects and their possible relation to honest status signaling in the Brownheaded Cowbird. The Condor 89: 1-23.

Rozzi, R., Martínez, D., Willson, M.F., Sabag, C. 1996. Avifauna de los bosques templados de Sudamérica. In: Armesto, J.J., Villagrán, C., Kalin, M.T. (Eds) Ecología de los bosques nativos de Chile: 135-152. Editorial Universitaria, Santiago, Chile.

Sandoval, L., Menill, D.J. 2014. A quantitative description of vocalizations and vocal behaviour of the Rustycrowned Ground-Sparrow (Melozone kieneri). Ornitología Neotropical 25: 219-230.

Sandoval, L., Méndez, C., Menill, D.J. 2014. Individual distinctiveness in the fine structural features and repertoire characteristics of the songs of White-eared Ground-sparrows. Ethology 120: 275-286.

Seddon, N. 2005. Ecological adaptation and species recognition drives vocal evolution in Neotropical suboscine birds. Evolution 59: 200-215.

Seddon, N., Tobias, J.A. 2007. Song divergence at the edge of Amazonia: An empirical test of the peripatric speciation model. Biological Journal of the Linnean Society 90: 173188.

Seddon, N., Tobias, J.A. 2010. Character displacement from the receiver's perspective: Species and mate recognition despite convergent signals in suboscine birds. Proceedings of the Royal Society of London, Series B 277: 2475-2483.

Seddon, N., Tobias, J.A., Alvarez, A. 2002. Vocal communication in the Pale-winged Trumpeter (Psophia leucoptera): Repertoire, context and functional reference. Behaviour 139: 1331-1359

Sedgwick, J.A. 2001. Geographic variation in the song of Willow 
Flycatchers: Differentiation between Empidonax traillii adastus and E. t. extimus. The Auk 118: 366-379.

Sibley, C.G., Alquist, J.E. 1990. Phylogeny and classification of birds. Yale University Press, New Haven, CT.

van Strien, M.J., Holderegger, R., Van Heck, H.J. 2015. Isolationby-distance in landscapes: Considerations for landscape genetics. Heredity 114(1): 27-37.

Toews, D.P., Irwin, D.E. 2008. Cryptic speciation in a Holarctic passerine revealed by genetic and bioacoustic analyses. Molecular Ecology 17: 2691-2705.

Tubaro, P.L., Segura, E.T. 1994. Dialect differences in the song of Zonotrichia capensis in the southern pampas - a test of the acoustic adaptation hypothesis. The Condor 96: 1084-1088.

Wilczynski, W., Ryan, M.J. 1999. Geographic variation in animal communication systems. In: Foster, S.A., Endler, J.A. (Eds) Geographic variation in behavior: 234-261. Oxford University Press.

Wilkins, M.R., Seddon, N., Safran, R.J. 2013. Evolutionary divergence in acoustic signals: Causes and consequences. Trends in Ecology and Evolution 28(3): 156-166.

Willson, M.F. 2004. Loss of habitat connectivity hinders pair formation and juvenile dispersal of Chucao tapaculos in Chilean rainforest. The Condor 106: 166-171.

APÉNDICE 1. Lista de grabaciones del chucao (Scelorchilus rubecula) disponibles en xeno-canto utilizadas para los análisis. Los archivos en formato .WAV están disponibles para solicitud en base de cantos de los autores. / List of recordings of Chucao Tapaculo (Scelorchilus rubecula) used for the analyzes available in xeno-canto database. Files in .WAV format are available upon request.

\begin{tabular}{ccccc}
\hline Repositorio & Número & Grabador & País & Localidad \\
\hline XC & 102707 & F. Schmitt & Chile & Altos de Lircay \\
XC & 218159 & L. Benner & Chile & Altos de Lircay \\
XC & 17875 & D. Knapp & Chile & Altos de Lircay \\
XC & 972 & R. Valenzuela & Chile & Altos de Lircay \\
XC & 102382 & J. King & Chile & Altos de Lircay \\
XC & 102380 & J. King & Chile & Altos de Lircay \\
XC & 60160 & A. Jaramillo & Chile & PN Conguillío \\
XC & 364090 & H.V. Norambuena & Chile & PN Conguillío \\
XC & 364093 & H.V. Norambuena & Chile & PN Conguillío \\
XC & 505950 & H.V. Norambuena & Chile & PN Conguillío \\
XC & 127698 & T. Tumiel & Argentina & PN Lago Puelo \\
XC & 60158 & A. Jaramillo & Chile & Nevados Chillán \\
XC & 60157 & A. Jaramillo & Chile & Nevados Chillán \\
XC & 50057 & B. López-Lanus & Argentina & PN Lanín \\
XC & 50058 & B. López-Lanus & Argentina & PN Lanín \\
XC & 52122 & B. López-Lanus & Argentina & PN Lanín \\
XC & 52121 & B. López-Lanus & Argentina & PN Lanín \\
\hline
\end{tabular}

Received: 26.02.2018

Accepted: 24.12.2019 